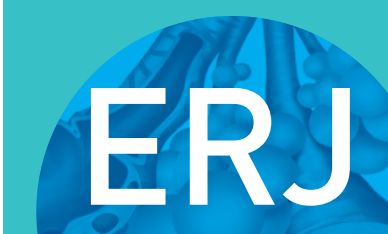

open research
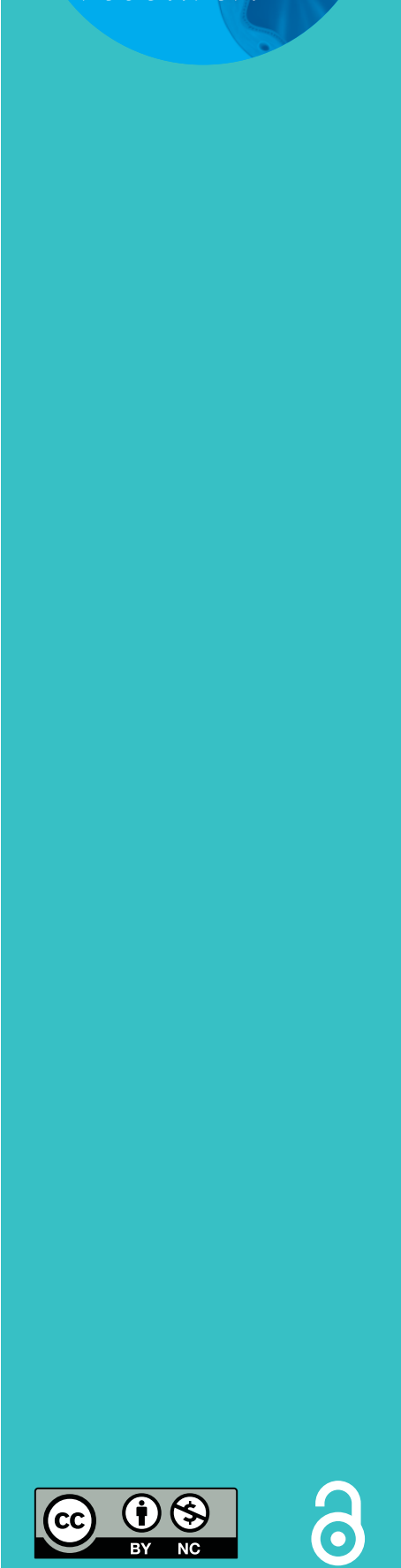

\section{Cystic fibrosis airway microbiota associated with outcomes of nontuberculous mycobacterial infection}

\author{
Lindsay J. Caverly', Madsen Zimbric (1) ${ }^{1}$, Michelle Azar (1) ${ }^{1}$, Kristopher Opron² \\ and John J. LiPuma ${ }^{1}$
}

Affiliations: ${ }^{1}$ Dept of Pediatrics, University of Michigan Medical School, Ann Arbor, MI, USA. ${ }^{2}$ Dept of Internal Medicine, University of Michigan Medical School, Ann Arbor, MI, USA.

Correspondence: Lindsay J. Caverly, L2221 UH South, 1500 E. Medical Center Dr., Ann Arbor, MI 48109, USA. E-mail: caverlylamed.umich.edu

\section{ABSTRACT}

Rationale: Pulmonary infections with nontuberculous mycobacteria (NTM) are increasingly prevalent in people with cystic fibrosis (CF). Clinical outcomes following NTM acquisition are highly variable, ranging from transient self-resolving infection to NTM pulmonary disease associated with significant morbidity. Relationships between airway microbiota and variability of NTM outcomes in CF are unclear.

Objective: To identify features of CF airway microbiota associated with outcomes of NTM infection.

Methods: 188 sputum samples, obtained from 24 subjects with $\mathrm{CF}$, each with three or more samples collected from 3.5 years prior to, and up to 6 months following incident NTM infection, were selected from a sample repository. Sputum DNA underwent bacterial $16 \mathrm{~S}$ rRNA gene sequencing. Airway microbiota were compared based on the primary outcome, a diagnosis of NTM pulmonary disease, using Wilcoxon rank-sum testing, autoregressive integrated moving average modelling and network analyses.

Measurements and main results: Subjects with and without NTM pulmonary disease were similar in clinical characteristics, including age and lung function at the time of incident NTM infection. Time-series analyses of sputum samples prior to incident NTM infection identified positive correlations between Pseudomonas, Streptococcus, Veillonella, Prevotella and Rothia with diagnosis of NTM pulmonary disease and with persistent NTM infection. Network analyses identified differences in clustering of taxa between subjects with and without NTM pulmonary disease, and between subjects with persistent versus transient NTM infection.

Conclusions: CF airway microbiota prior to incident NTM infection are associated with subsequent outcomes, including diagnosis of NTM pulmonary disease, and persistence of NTM infection. Associations between airway microbiota and NTM outcomes represent targets for validation as predictive markers and for future therapies.

@ERSpublications

In patients with CF and NTM infection, structure and dynamics of airway microbiota preceding NTM infection are associated with subsequent, clinically relevant NTM infection outcomes, including NTM pulmonary disease and persistence of NTM infection https://bit.ly/36JVAGW

Cite this article as: Caverly LJ, Zimbric M, Azar M, et al. Cystic fibrosis airway microbiota associated with outcomes of nontuberculous mycobacterial infection. ERJ Open Res 2021; 7: 005782020 [https://doi.org/10.1183/23120541.00578-2020].

This article has supplementary material available from openres.ersjournals.com

Received: 13 Aug 2020 | Accepted after revision: 29 Jan 2021

Copyright $\odot$ The authors 2021. This version is distributed under the terms of the Creative Commons Attribution NonCommercial Licence 4.0. For commercial reproduction rights and permissions contact permissions@ersnet.org 


\section{Introduction}

Nontuberculous mycobacterial (NTM) infections, particularly with Mycobacterium avium complex and Mycobacterium abscessus complex species, affect approximately one-quarter of people with cystic fibrosis $(\mathrm{CF})$, and are increasing in prevalence $[1,2]$. Outcomes of NTM infection in CF are highly variable, resulting in NTM pulmonary disease [3,4] (i.e. clinical decline attributed to NTM infection) in approximately one-third of cases [5]. In the other two-thirds, NTM infection is either transient, or persistent in the airways without associated clinical decline [5]. Mechanisms underlying the variability in clinical outcomes of NTM infection are unclear, and predictors of NTM pulmonary disease at the time of initial NTM infection are lacking.

In non-CF populations, culture-independent studies of respiratory samples have identified positive associations between certain oral-associated microbiota (e.g. Prevotella) and NTM infection [6, 7], as well as positive associations between the relative abundance of Prevotella and adverse outcomes of NTM infection, including chest imaging abnormalities [6] and levels of inflammation [7]. One proposed mechanism for oral-associated microbiota influencing susceptibility to NTM infection includes inhibition of host response to mycobacteria by short-chain fatty acids produced through anaerobic metabolism [8]. While the role of oral-associated microbiota in NTM infection in CF are unclear, these taxa are prevalent in CF airways [9], and are associated with adverse outcomes, including pulmonary exacerbation [10, 11] and inflammation $[12,13]$ in (non-NTM infected) people with CF.

We hypothesised that oral-associated microbiota in $\mathrm{CF}$ airways are related to the variability of clinical outcomes of NTM infection in people with CF. To test this hypothesis, we performed a retrospective medical record review and analysed CF sputum samples that had been collected prior to incident NTM infection to identify features of airway microbiota associated with clinical and microbiological outcomes within the first year following infection.

\section{Methods}

\section{Subjects and sputum sample repository}

Under approval from the Michigan Medicine institutional review board, sputum samples had been previously collected through the course of routine clinical care in the years $2000-2015$, and stored at $-80^{\circ} \mathrm{C}$ until the time of DNA extraction. Subjects were selected who had a diagnosis of CF, and from whom three or more sputum samples were available that had been collected prior to, and up until 6 months following, their incident NTM infection (i.e. their first NTM-positive culture). Subjects were excluded if their incident NTM infection occurred following organ transplantation or if the only NTM species identified was Mycobacterium gordonae.

In order to assess outcomes following incident NTM infection, clinical data, including culture data for acid-fast bacilli (AFB) and CF pathogens, during 1 year prior to and 1 year following incident NTM infection were extracted from the Michigan Medicine electronic medical record. Cultures for AFB and CF pathogens were performed by the Michigan Medicine clinical microbiology laboratory. The clinical variables selected were those known to be associated with differences in airway microbiota and/or severity of CF lung disease (see additional details in the supplementary methods). The primary (clinical) outcome was a physician-defined diagnosis of NTM pulmonary disease (i.e. physician determined that American Thoracic Society/Infectious Diseases Society of America clinical, microbiologic and/or radiologic criteria for NTM pulmonary disease $[3,4]$ were met, and initiated antimycobacterial treatment) within the year following incident NTM infection. In each case, the physician diagnosis of NTM pulmonary disease was confirmed through review of provider documentation and verification of prescription of antimycobacterial treatment. Secondary (microbiological) outcomes were persistent NTM infection (defined as at least two respiratory cultures positive for the same NTM species within 1 year following incident NTM infection, whether or not NTM pulmonary disease was diagnosed) or transient NTM infection (defined as a single respiratory culture positive for NTM, with at least one subsequent negative AFB culture) [5].

\section{Sputum microbial community measures}

Full methods are included in the supplementary material. In brief, stored sputum samples were thawed on ice, homogenised and DNA was extracted as described previously [14]. Sequencing of the V4 region of the bacterial 16S rRNA gene was performed by the University of Michigan Microbial Systems Molecular Biology Laboratory as described previously [15]. Mock community DNA was sequenced to determine sequencing error rates. Water controls were included to assess for PCR contamination, and reagent controls were sequenced to assess for DNA contamination of sputum samples. Raw sequencing data are available at the National Center for Biotechnology Information (BioProject PRJNA594304).

The raw DNA sequences were processed using mothur (1.40.4) [16] and the MiSeq standard operating procedure (accessed 17 October 2018). Briefly, after discarding low-quality and chimeric sequences, 
sequences were assigned to taxonomy against the SILVA database (release 132) using the Ribosomal Database Project Bayesian classifier and clustered into operational taxonomic units (OTUs) based on $97 \%$ similarity using the OptiClust algorithm [17]. Data were rarified for calculation of $\alpha$-diversity measures.

Total bacterial load was quantified on all sputum samples and reagent control DNA extractions using droplet digital PCR (ddPCR; Bio-Rad QX200 AutoDG Droplet Digital PCR System, Bio-Rad, Hercules, CA, USA) with primers and a modified probe targeting conserved regions of the bacterial 16S rRNA gene [18]. Full ddPCR methods are included in the supplementary material.

\section{Data analyses}

Clinical and demographic data were compared between subjects with and without NTM disease. p-values were calculated with Boschloo's exact test in the R package "exact" for binomial variables [19], the Fisher-Freeman-Halton test with mid-p correction from the $\mathrm{R}$ package "epitools" for nominal variables [20], and Wilcoxon's rank-sum test in base $\mathrm{R}$ for numeric variables [21]. The base $\mathrm{R}$ wilcox.test function was used for Wilcoxon rank-sum testing of microbiome features (cross-sectional and longitudinal relative abundances of OTUs, $\alpha$-diversity measures and total bacterial load) and percentage predicted forced expiratory volume in $1 \mathrm{~s}\left(\mathrm{ppFEV}_{1}\right)$ between NTM outcome groups.

Time-series data of OTU relative abundance values were analysed with an autoregressive integrated moving averages (ARIMA) model as described by RiDENHOUR et al. [22]. In brief, this particular ARIMA model uses a Poisson regression to estimate interactions between taxa in time-series data. The correlation matrices for the ARIMA models were made with the R package "corrplot". The network graphs were generated from the ARIMA model outputs, using "igraph" in R. Full ARIMA methods are included in the supplementary material.

Sputum DNA extraction protocol, mothur command file, ddPCR data, de-identified clinical data, sample IDs and OTU table and code for analyses and figures are available at https://github.com/caverlyl/CF_ NTM_microbiome/.

\section{Results}

\section{Subject clinical features}

24 subjects from whom three or more sputum samples $(n=188)$ were available were included. A mean of 7.8 samples per subject (range 3-19 samples per subject) were available for study, and samples ranged from 3.5 years prior to, and up to 6 months following incident NTM infection (supplementary figure S1). The number of samples per subject, and the clinical state of the subjects (defined in the supplementary methods) at the times of sample collection did not differ between subjects in whom NTM pulmonary disease ultimately was or was not diagnosed (table 1).

Eight $(33 \%)$ of the 24 subjects were ultimately diagnosed with NTM pulmonary disease and started on antimycobacterial antibiotics within 1 year of incident NTM infection. Overall, at the time of incident infection, subjects subsequently diagnosed with NTM pulmonary disease did not differ in age, lung function $\left(\mathrm{ppFEV}_{1}\right)$, prevalence of CF pathogens in respiratory cultures and use of chronic (i.e. thrice weekly oral azithromycin or alternating months of inhaled antibiotics) or episodic (i.e. antibiotic course prescribed to treat pulmonary exacerbation) antibiotics compared to those who were not subsequently diagnosed with NTM pulmonary disease (table 2). None of the subjects had multiple NTM species present

\begin{tabular}{|c|c|c|c|}
\hline & $\begin{array}{c}\text { No NTM pulmonary } \\
\text { disease }\end{array}$ & $\begin{array}{c}\text { NTM pulmonary } \\
\text { disease }\end{array}$ & p-value \\
\hline Subjects & 16 & 8 & \\
\hline $\begin{array}{l}\text { Total sputum samples (mean per } \\
\text { subject, range) }\end{array}$ & $129(8.1,3-19)$ & $59(7.4,3-14)$ & 0.73 (unpaired t-test) \\
\hline \multicolumn{4}{|l|}{ Sample clinical state ${ }^{\#}$} \\
\hline Baseline & 30 (23.3) & 22 (37.3) & 0.06 (Chi-squared test) \\
\hline Exacerbation & $37(28.7)$ & 18 (30.5) & \\
\hline Treatment & $42(32.6)$ & 9 (15.3) & \\
\hline Recovery & 20 (15.5) & 10 (16.9) & \\
\hline
\end{tabular}




\section{TABLE 2 Subject demographics and clinical characteristics}

\begin{tabular}{|c|c|c|c|}
\hline & $\begin{array}{c}\text { No NTM pulmonary } \\
\text { disease }\end{array}$ & $\begin{array}{c}\text { NTM pulmonary } \\
\text { disease }\end{array}$ & p-value \\
\hline Subjects & 16 & 8 & \\
\hline Age years ${ }^{\#}$ & $27.3(21.9-37.7)$ & $25.8(19.7-32.7)$ & 0.83 \\
\hline Male & $9(56.2)$ & $2(25)$ & 0.15 \\
\hline \multicolumn{4}{|l|}{ CF genotype } \\
\hline F508del homozygous & 4 (25) & 3 (37.5) & \multirow[t]{2}{*}{0.49} \\
\hline F508del heterozygous & $11(68.8)$ & $4(50)$ & \\
\hline Other & $1(6.3)$ & $1(12.5)$ & 0.59 \\
\hline \multicolumn{4}{|l|}{ NTM species } \\
\hline M. avium complex & $9(56.2)$ & $6(75)$ & \multirow[t]{2}{*}{0.81} \\
\hline M. abscessus complex & $4(25)$ & $2(25)$ & \\
\hline Other & $3(18.6)$ & $0(0)$ & 0.42 \\
\hline AFB smear positive ${ }^{\#}$ & $2(12.5)$ & $2(25)$ & 0.44 \\
\hline ppFEV $_{1}^{\#}$ & $55(37-69)$ & $68(43-80)$ & 0.41 \\
\hline \multicolumn{4}{|l|}{ Disease aggressiveness ${ }^{\eta,+}$} \\
\hline Mild & $9(56.2)$ & 3 (37.5) & \\
\hline Moderate & $4(25)$ & 3 (37.5) & 0.47 \\
\hline Severe & $3(18.6)$ & $2(25)$ & 0.59 \\
\hline \multicolumn{4}{|l|}{$\mathrm{BMI}^{\#,+}$} \\
\hline Acceptable & $4(40)$ & 1 (33.3) & \\
\hline At risk & $2(20)$ & 1 (33.3) & 0.75 \\
\hline Nutritional failure & $4(40)$ & 1 (33.3) & 1 \\
\hline \multicolumn{4}{|l|}{ CF respiratory cultures $\#$} \\
\hline$P$. aeruginosa & 9 (56.2) & $6(75)$ & 0.55 \\
\hline S. aureus & 14 (87.5) & 3 (37.5) & 0.01 \\
\hline S. maltophilia & $3(18.6)$ & $0(0)$ & 0.38 \\
\hline Achromobacter spp. & 2 (12.5) & $1(12.5)$ & 1 \\
\hline Burkholderia spp. & $0(0)$ & $0(0)$ & \\
\hline Aspergillus spp. & 5 (31.3) & $2(25)$ & 1 \\
\hline \multicolumn{4}{|l|}{ CF respiratory cultures, $\geqslant 1$ positive } \\
\hline P. aeruginosa & $10(62.5)$ & $6(75)$ & 0.57 \\
\hline S. aureus & $16(100)$ & $6(75)$ & 0.07 \\
\hline S. maltophilia & $3(18.6)$ & $1(12.5)$ & 1 \\
\hline Achromobacter spp. & $3(18.6)$ & $1(12.5)$ & 1 \\
\hline Burkholderia spp. & $1(6.3)$ & $0(0)$ & 1 \\
\hline Aspergillus spp. & 6 (37.5) & $6(75)$ & 0.13 \\
\hline Diagnosis of CF-related diabetes" & $5(31.3)$ & $4(50)$ & 0.33 \\
\hline Pulmonary exacerbations $s^{\eta,+}$ & $2(0.75-5)$ & $2(1.75-3.75)$ & 0.88 \\
\hline Use of chronic azithromycin & $9(56.2)$ & $5(62.5)$ & 1 \\
\hline Use of chronic inhaled antibiotics" & $10(62.5)$ & $6(75)$ & 0.57 \\
\hline Use of inhaled steroids & $10(62.5)$ & $8(100)$ & 0.04 \\
\hline \multicolumn{4}{|c|}{$\begin{array}{l}\text { Data are presented as } \mathrm{n} \text {, median (interquartile range) or } \mathrm{n}(\%) \text {, unless otherwise stated. NTM: } \\
\text { nontuberculous mycobacteria; CF: cystic fibrosis; M. avium: Mycobacterium avium; M. abscessus: } \\
\text { Mycobacterium abscessus; AFB: acid-fast bacilli; } \text { ppFEV }_{1} \text { : percentage predicted forced expiratory volume in } \\
1 \mathrm{~s} \text {; BMI: body mass index; P. aeruginosa: Pseudomonas aeruginosa; S. aureus: Staphylococcus aureus; } \\
\text { S. maltophilia: Stenotrophomonas maltophilia. }{ }^{\#} \text { : at incident NTM infection; }{ }^{\text {I }} \text { : within } 1 \text { year prior to incident } \\
\text { NTM infection; }{ }^{+} \text {: details in supplementary methods. }\end{array}$} \\
\hline
\end{tabular}

at the time of incident infection. During the study period, none of the subjects received cystic fibrosis transmembrane conductance regulator modulator therapy, and none of the subjects was diagnosed with allergic bronchopulmonary aspergillosis. Among the 23 subjects from whom follow-up AFB cultures were available to determine microbiological outcomes (supplementary figure S1), eight (35\%) had persistent NTM infection (six of these were diagnosed with NTM pulmonary disease), whereas 15 had transient NTM infection.

\section{DNA sequencing controls}

Sequencing error rates based on analyses of mock communities ranged from 0.000177 to 0.00413 . Total bacterial load of sputum sample DNA extractions was $\sim 10^{4}$ greater than the bacterial load of reagent control DNA extractions (supplementary figure S2). Rank abundance plots of reagent controls showed 
minimal overlap between the top OTUs found in reagent controls with the OTUs found in the sputum samples (supplementary figure S3).

Six of the AFB culture-positive samples from subjects at the time of incident NTM infection were available for study inclusion (supplementary figure S1). Only one of these AFB culture-positive samples (16.7\%) had OTUs identified as Mycobacteria; this sample was also the only smear-positive sample. Mycobacteria OTUs were additionally identified in five samples (that were either AFB culture negative, or AFB culture was not performed) from four subjects collected prior to their incident NTM infection. One of these five samples was collected within a month of the subject's incident NTM infection, and the other four were collected between 1 and 3 years prior to the subjects' incident NTM infection. When present, the relative abundance of Mycobacteria OTUs averaged 0.044\% (range 0.0028-0.14\%).

\section{Microbiome features associated with NTM outcomes}

Cross-sectional features of the microbiota in sputum samples obtained from all subjects closest in time to their incident NTM infection, and longitudinal bacterial community features (linear regression slopes) in serial samples from individuals were compared between subjects with and without NTM pulmonary disease, and between subjects with transient versus persistent NTM infection. Subjects subsequently diagnosed with NTM pulmonary disease had an overall increasing relative abundance of Rothia across serial samples leading up to their incident NTM infection (slope of regression line $>0$ ), whereas subjects not diagnosed with NTM pulmonary disease had an overall decreasing relative abundance of Rothia (slope of regression line $<0)(p=0.027$; figure 1a). Persistent NTM infection was significantly associated with a greater relative abundance of Rothia at the time of incident NTM infection ( $\mathrm{p}=0.005$; figure $1 \mathrm{~b}$ ). In addition, persistent NTM infection was significantly associated with greater relative abundances of Prevotella ( $\mathrm{p}=0.02$; figure $1 \mathrm{c}$ ) and Veillonella ( $\mathrm{p}=0.4$; figure $1 \mathrm{e})$ at the time of incident NTM infection, as well as an increasing relative abundance of Fusobacterium in the longitudinal samples ( $\mathrm{p}=0.02$; figure $1 \mathrm{~d}$ ). Complete results of Wilcoxon rank-sum testing for all features across both clinical and microbiological outcomes are listed in supplementary tables S1 and S2, respectively. Neither total bacterial load of samples, nor $\mathrm{ppFEV}_{1}$ differed significantly between either of the outcome groups.

To evaluate relationships between changes in OTU relative abundances over time leading up to incident NTM infection and subsequent NTM outcomes, the outcome variables (NTM pulmonary disease or persistent NTM infection) were first forced into their respective ARIMA models for each OTU. OTUs positively correlated with both NTM pulmonary disease and persistent NTM infection in these models included Pseudomonas, Streptococcus, Veillonella, Prevotella and Rothia (table 3). OTUs negatively correlated with both NTM pulmonary disease and persistent NTM infection included Staphylococcus, Gemella and Stenotrophomonas. The direction of the correlation coefficients was generally consistent across the two outcomes, with the notable exception of Achromobacter (OTU0010), which was positively associated with NTM disease, but negatively associated with persistent NTM infection. This difference in direction of the effect between the two NTM outcomes was related to the low prevalence of Achromobacter (table 2), but high relative abundance when present.

\section{Microbial community networks}

An ARIMA model that included all subjects and samples was first used to model the bacterial community network of the entire cohort (supplementary figure S4). This network revealed interactions between traditional CF pathogens (i.e. Pseudomonas, Staphylococcus, Stenotrophomonas, Achromobacter) and oral-associated taxa (e.g. Prevotella, Rothia, Veillonella, Streptococcus). ARIMA models were then run separately for each outcome group and visualised as microbial community networks (figures 2 and 3 ). Subjects with NTM pulmonary disease had more significant relationships between OTUs as evidenced by the increased number of nodes, and increased number of edges between node clusters in the network when compared to subjects without NTM pulmonary disease (modularity scores of 0.169 and 0.182 , respectively). Similarly, subjects with persistent NTM infection had increased number of nodes, and increased number of edges between node clusters in the network when compared to subjects with transient NTM infection (modularity scores of 0.111 and 0.293 , respectively). The increased taxa connectedness observed in subjects with NTM pulmonary disease and in subjects with persistent NTM infection suggest that these NTM outcomes occur in subjects with less stable microbial communities, in which perturbing one OTU is more likely to affect other community members.

\section{Discussion}

The causes of the observed variability in clinical course of NTM infection in people with CF, with approximately one-third of individuals receiving a subsequent diagnosis of NTM pulmonary disease [5], are largely unknown, and predictors of NTM pulmonary disease are lacking. In this study, we observed significant differences in microbiota in sputum samples obtained prior to incident NTM infection between 

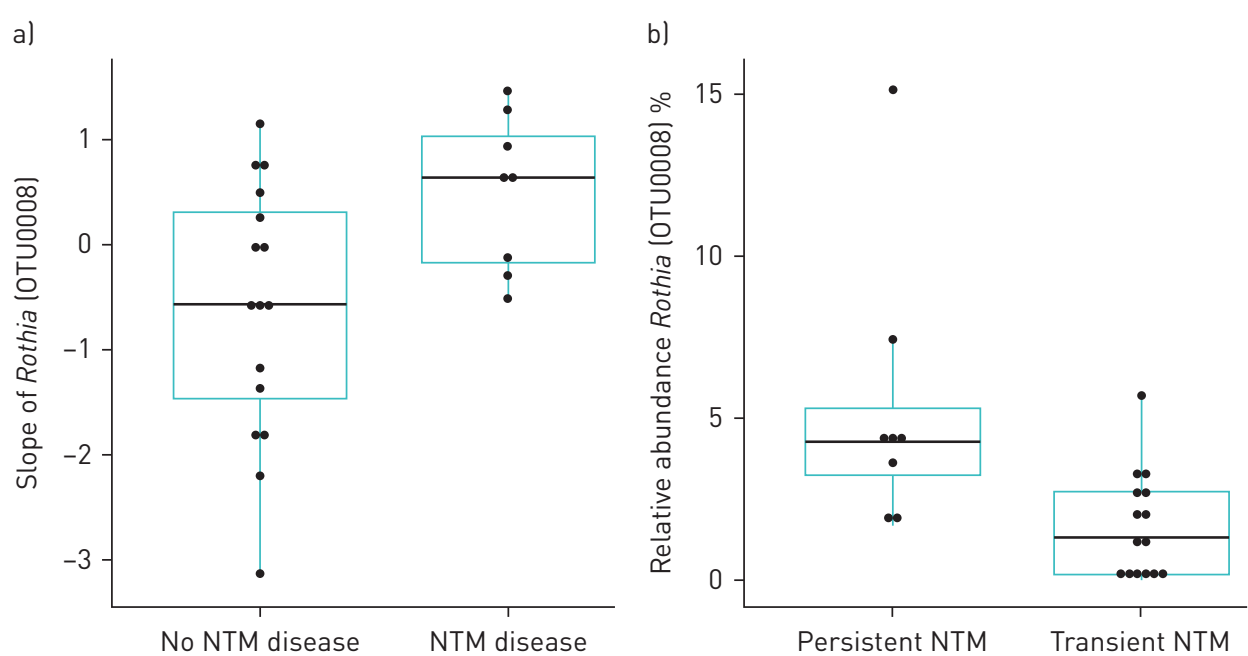

Persistent NTM

Transient NTM

e)

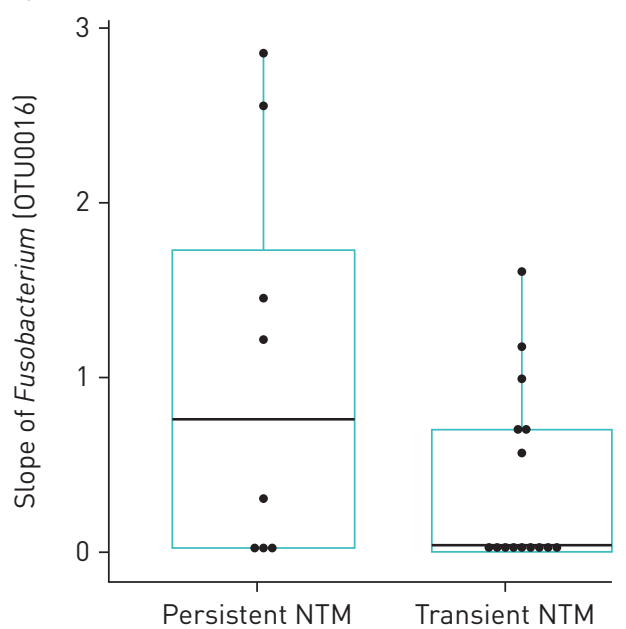

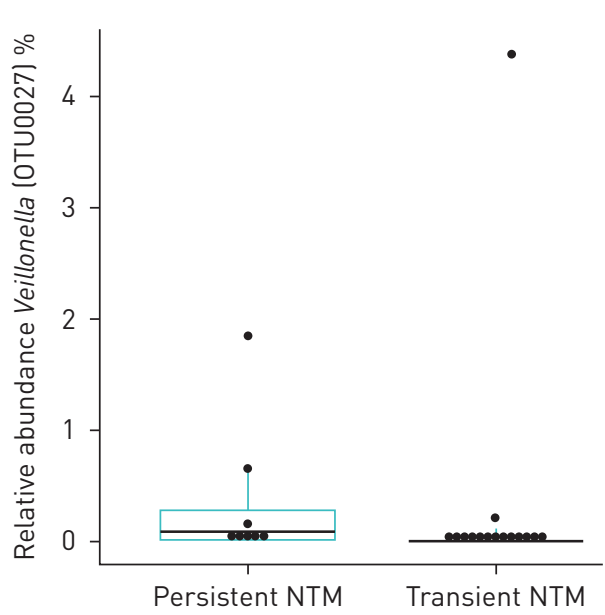

Persistent NTM Transient NTM
Persistent NTM Transient NTM

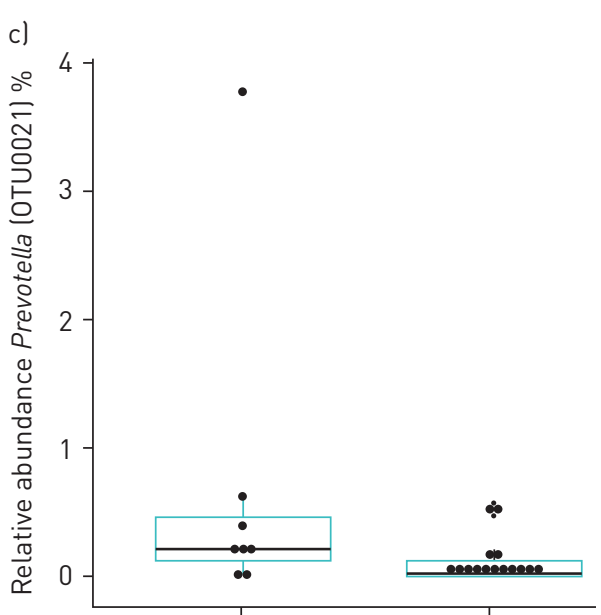

Persistent NTM Transient NTM

FIGURE 1 Microbial features significantly associated with either a) clinical (nontuberculous mycobacteria (NTM) pulmonary disease) or b-e) microbiological (transient versus persistent infection) NTM outcomes. Operational taxonomic units (OTUs) are labelled at genus level, and boxplots show the median and interquartile range. All p-values<0.05, Wilcoxon rank-sum 
TABLE 3 Autoregressive integrated moving averages (ARIMA) correlation coefficients of clinical and microbiological nontuberculous mycobacteria (NTM) outcomes with all operational taxonomic units (OTUs) included in final ARIMA model. The colours indicate the magnitude and direction of the effect of the outcomes on the OTUs, with the maximum negative effect $(-1.79)$ in red, the maximum positive effect (1.14) in blue, and no effect in white.

\begin{tabular}{llcc}
\hline & Genus & NTM pulmonary disease & Persistent NTM infection \\
\hline OTU0001 & Staphylococcus & -0.47 & -0.18 \\
OTU0002 & Pseudomonas & 1.06 & 0.78 \\
OTU0003 & Streptococcus & 0.31 & 0.06 \\
OTU0004 & Veillonella & 1.01 & 0.78 \\
OTU0005 & Prevotella & 0.79 & 0.38 \\
OTU0006 & Streptococcus & 0.36 & 0.21 \\
OTU0007 & Streptococcus & -0.02 & 0.02 \\
OTU0008 & Rothia & 0.75 & 0.54 \\
0TU0009 & Haemophilus & -0.15 & 0.08 \\
OTU0010 & Achromobacter & 1.14 & -1.79 \\
OTU0011 & Lactobacillales_unclassified & & 0.49 \\
0TU0012 & Neisseria & 0.63 & 0.10 \\
0TU0013 & Gemella & -0.05 & -0.13 \\
0TU0014 & Stenotrophomonas & -0.06 & -0.06 \\
0TU0015 & Actinomyces & 0.01 & 0.07 \\
OTU0023 & Prevotella & 0.91 & 1.07 \\
\hline
\end{tabular}

subject groups based on subsequent clinical and microbiological outcomes. More specifically, time-series modelling of samples from periods leading up to incident NTM infection identified associations between increasing relative abundances of certain taxa, including Pseudomonas, Streptococcus, Veillonella, Prevotella and Rothia, with a subsequent diagnosis of NTM pulmonary disease, and with persistent NTM infection. Negative correlations between certain taxa, including Staphylococcus, Gemella and Stenotrophomonas and a subsequent diagnosis of NTM pulmonary disease, and with persistent NTM infection, were also observed, and generate further hypotheses regarding relationships between microbial community dynamics and NTM infection outcomes.

When these time-series (ARIMA) models of bacterial community dynamics leading up to incident NTM infection were visualised as bacterial community networks, differences in network topologies were associated with NTM outcomes. Networks from subjects with NTM pulmonary disease, and persistent NTM infection, had increased numbers of nodes (i.e. genus-level OTU groupings) and edges between nodes compared to patients without NTM pulmonary disease, and with transient NTM infection, respectively. Based on ecological network theory, more tightly connected communities (as observed in patients with NTM pulmonary disease, and persistent NTM infection) may be more fragile, as perturbing one OTU is more likely to affect other community members [23, 24]. In CF airways, re-organisation of bacterial community networks to a pattern of increased numbers of nodes and edges has been associated with the transition from baseline clinical state to pulmonary exacerbation [25]. Our ability to identify direct network interactions between NTM and other OTUs was limited by the poor sensitivity of $16 \mathrm{~S}$ rRNA gene sequencing for NTM identification, consistent with prior work [7, 14], but will be of interest for future study.

These findings offer novel insight into airway bacterial community members, dynamics and interactions as potential contributors to the variability in outcomes observed with NTM infection in people with CF. Testing of hypotheses informed by these observations will seek to define the host and microbial mechanisms underlying these relationships. Our results are consistent with studies in non-CF bronchiectasis, in which the relative abundances of certain anaerobic species have been found to be positively associated with degrees of chest CT abnormalities [6] and levels of inflammation [7]. However, such studies have been limited to patients with existing NTM infection. The lack of samples preceding NTM infection limits insight into whether airway microbiota contribute to NTM infection or merely reflect the consequences of NTM-related modulation of airway bacterial communities [26]. In this regard, the availability of sputum samples collected prior to, and at the time of, incident NTM infection in our study adds a new temporal component to the associations between airway microbiota and outcomes of NTM infection. 
a)

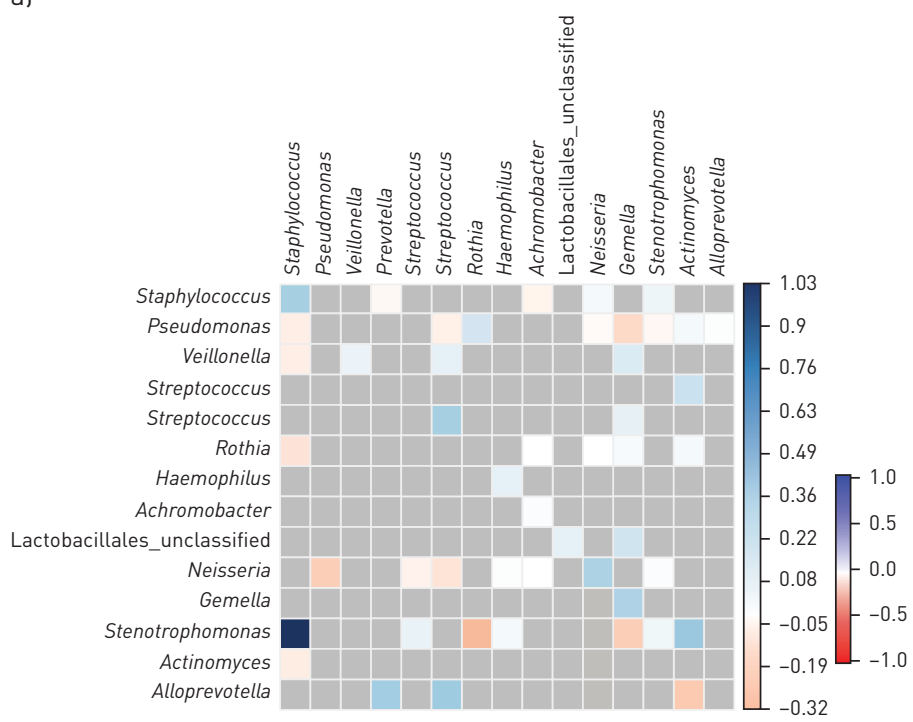

c)

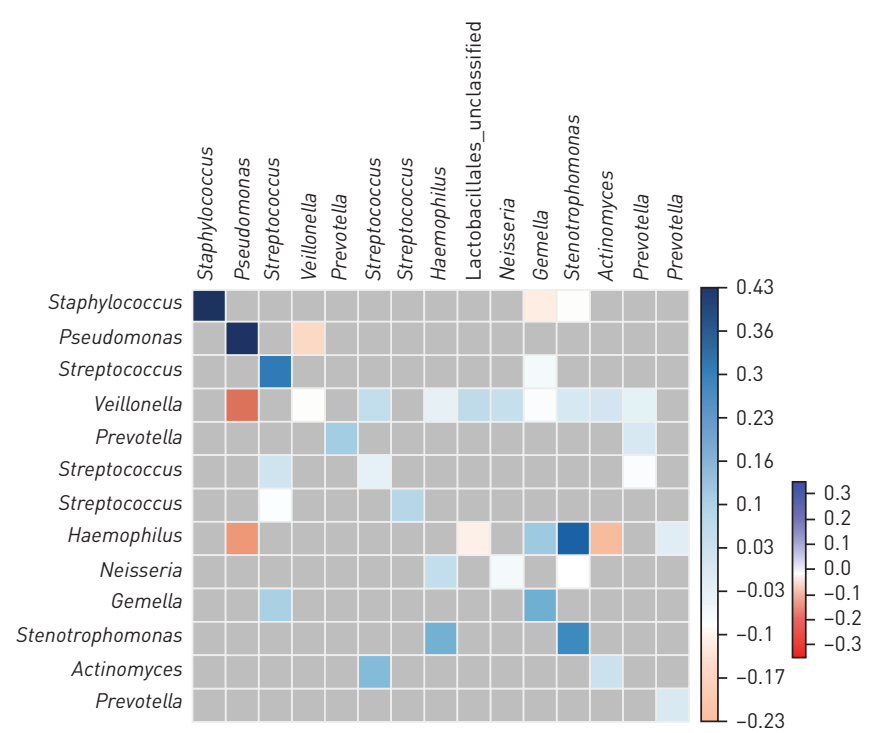

b)

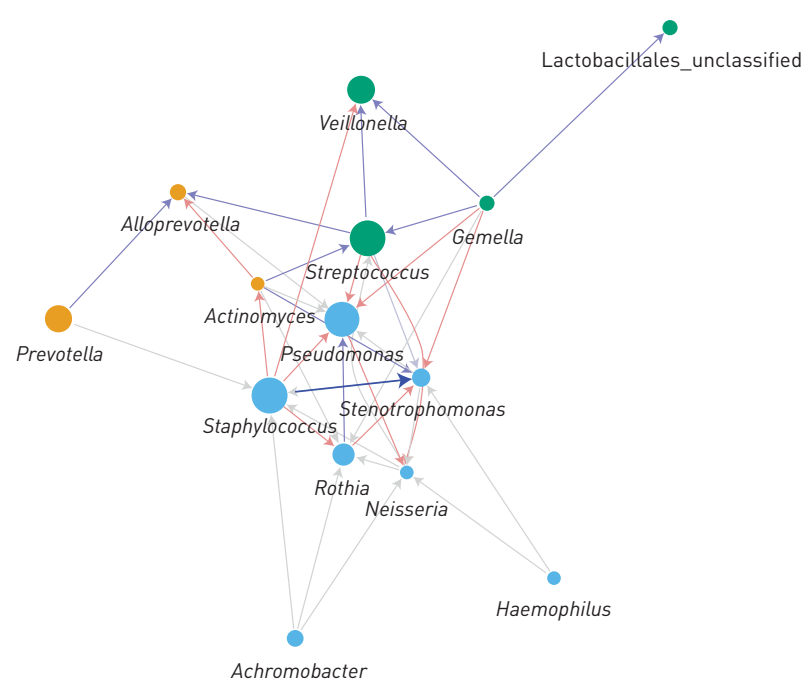

d)

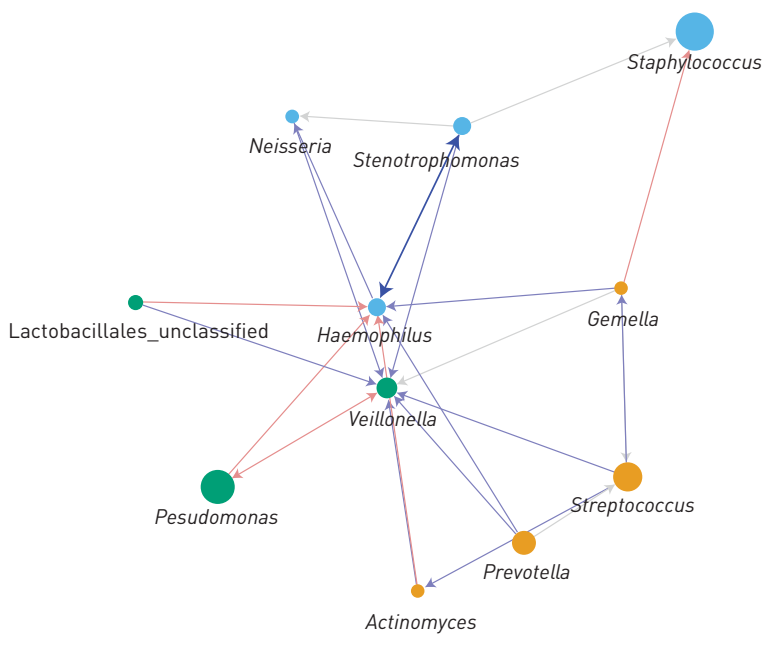

FIGURE 2 Differences in bacterial community interactions between cohorts with and without nontuberculous mycobacteria (NTM) pulmonary disease. Heat maps of autoregressive integrated moving averages (ARIMA) model show mean pairwise effect sizes of interacting operational taxonomic units (OTUs) in subjects a) with and c) without NTM pulmonary disease. In the heat map, OTUs on the horizontal axis affect the OTUs on the vertical axis. The heat map colours indicate the magnitude and direction of the OTU interactions (positive interactions in blue, negative interactions in red). In the network graphs of subjects b) with and d) without NTM pulmonary disease, each vertex represents the genus-level grouping of all OTUs included in that ARIMA model. Vertices are coloured by cluster, and are sized by OTU relative abundance. The edges are coloured according to their ARIMA correlation coefficients as shown on the heat maps. Arrows indicate the directionality of the network relationships, i.e. the predicting genus points toward the predicted genus. Due to the asymmetric nature of the ARIMA correlation matrix and the genus-level grouping of OTUs, multiple edges between each pair of vertices are possible.

Based on guidelines for the management of NTM infection in CF, incident infection typically prompts antibiotic treatment that is directed against other co-infecting bacterial pathogens. In fact, response to therapy targeting co-pathogens is included as a criterion for determining whether NTM infection is contributing to pulmonary disease (i.e. NTM pulmonary disease) $[3,27]$. The sputum samples used in our study were collected prior to the initiation of intensified antibiotic treatment of CF co-pathogens, as well as any NTM-directed antibiotics, thereby avoiding confounding of our results by the impact of intensive antibiotic treatment on airway microbiota.

To further address potential confounding by variables that influence airway microbiota, we evaluated differences in clinical state [10] at the time of sputum sample collection between subjects subsequently diagnosed with or without NTM pulmonary disease. While there was a trend towards a greater percentage of samples collected at baseline clinical state, with fewer samples collected during the time of antibiotic 
a)

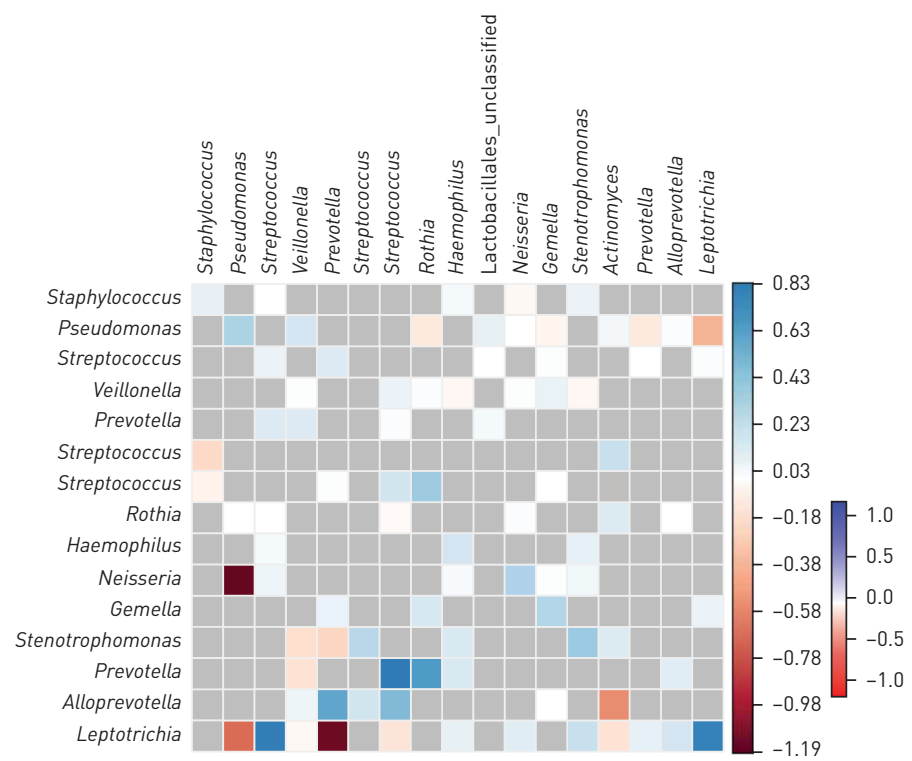

c)

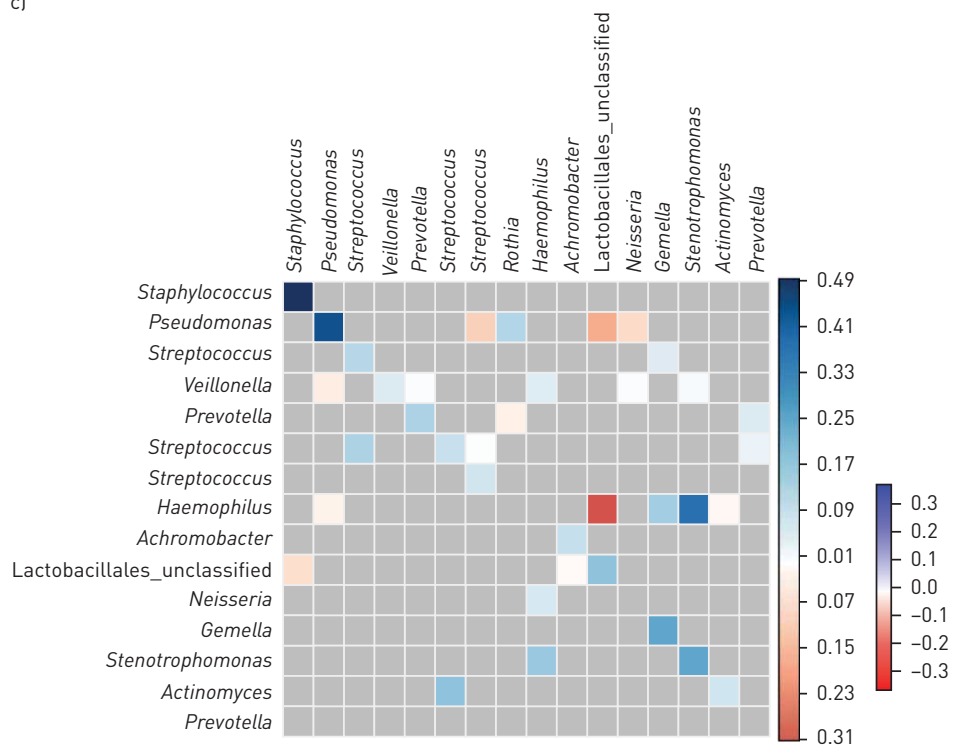

b
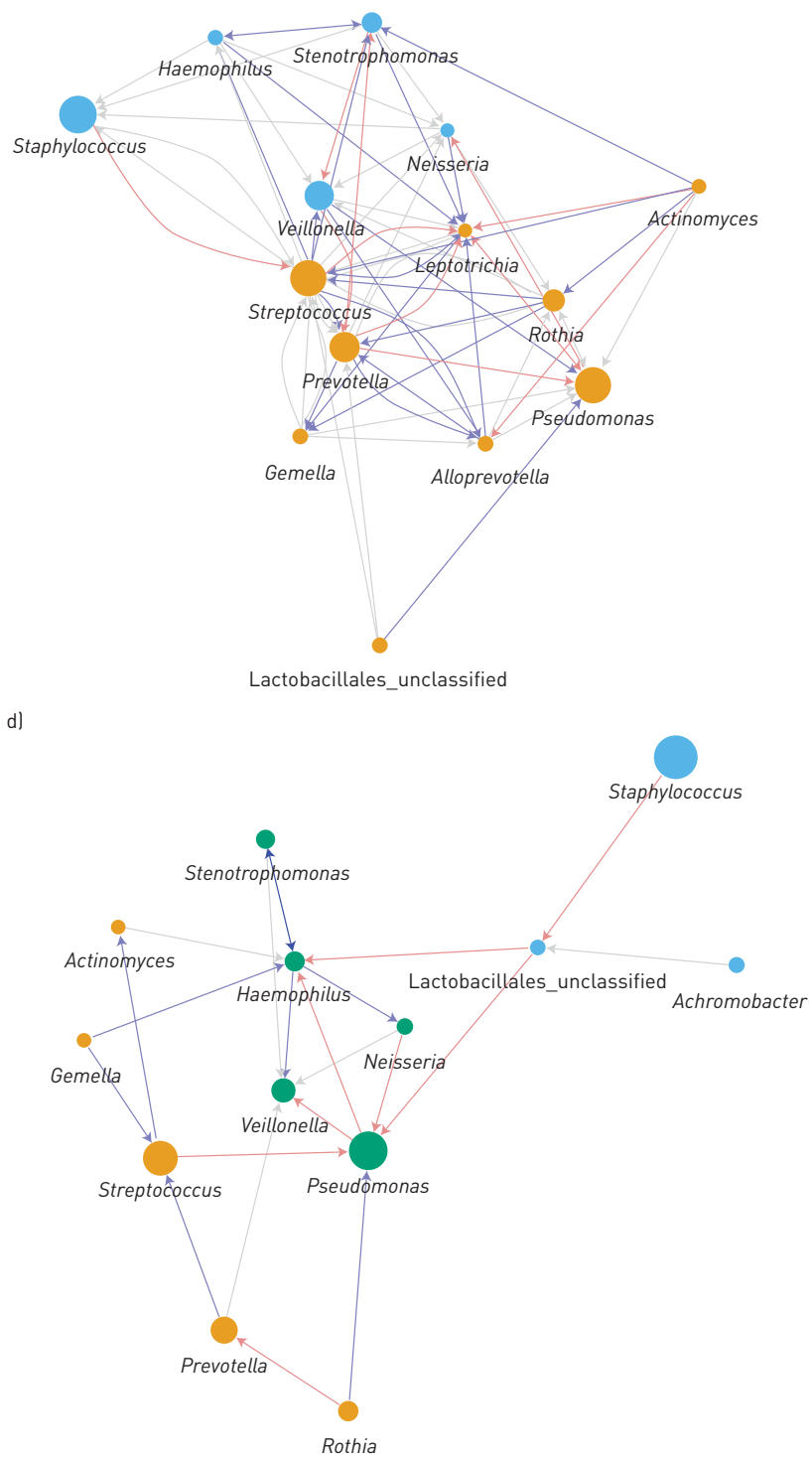

FIGURE 3 Differences in bacterial community interactions between cohorts with persistent compared to transient nontuberculous mycobacteria (NTM) infection. Heat maps of autoregressive integrated moving averages (ARIMA) model show mean pairwise effect sizes of interacting operational taxonomic units (OTUs) in subjects with a) persistent and c) transient NTM infection. In the heat map, OTUs on the horizontal axis affect the OTUs on the vertical axis. The heat map colours indicate the magnitude and direction of the OTU interactions (positive interactions in blue, negative interactions in red). In the network graphs of subjects with b) persistent and d) transient NTM infection, each vertex represents the genus-level grouping of all OTUs included in that ARIMA model. Vertices are coloured by cluster, and are sized by OTU relative abundance. The edges are coloured according to their ARIMA correlation coefficients as shown on the heat maps. Arrows indicate the directionality of the network relationships, i.e. the predicting genus points toward the predicted genus.

treatment, in subjects subsequently diagnosed with NTM pulmonary disease compared to those without, clinical state at the time of sample collection did not significantly differ between the groups. Furthermore, subject groups with and without NTM pulmonary disease did not differ in multiple additional clinical features known to be associated with differences in airway microbiota, (e.g. age, $\mathrm{ppFEV}_{1}$, disease stage, $\mathrm{CF}$ pathogens on bacterial culture, use of inhaled antibiotics, number of pulmonary exacerbations) [28-31]. While sample size limited our ability to control for all of the clinical variables relevant to measures of airway microbiota, and we cannot rule out variability in these data measurements over time (e.g. pulmonary function testing methodology), the lack of differences in these features between outcome groups supports the hypothesis that the observed differences in airway microbiota preceding and at the time of NTM acquisition have bearing on subsequent NTM outcomes, rather than representing confounding variables. We did observe higher rates of inhaled steroid use in the subjects with NTM 
pulmonary disease, and cannot rule out the potential that this may have impacted the bacterial community measures.

Diagnosing NTM pulmonary disease in CF requires meeting both microbiological (two or more positive sputum NTM cultures, or one or more positive bronchoalveolar lavage or lung biopsy culture) and clinical criteria (signs and symptoms of clinical decline, despite optimisation of other CF-related comorbidities) $[3,4]$. The latter has inherent subjectivity, and may vary from provider to provider, and from patient to patient. Future development of more standardised approaches to the diagnosis of NTM pulmonary disease in CF, as is currently being tested in the Prospective Evaluation of NTM Disease in Cystic Fibrosis (PREDICT) study, are needed to address this limitation in future studies. To address the subjectivity in NTM pulmonary disease diagnosis, we used persistent NTM infection (versus transient NTM infection) as a secondary, more objective microbiological outcome to assess the association between airway microbiota and NTM disease. In the aggregate, we found significant differences in airway microbiota between individuals who had persistent NTM infection compared to those with transient infection. However, we note that among the eight subjects in our study cohort with persistent NTM infection, only six had physician-diagnosed NTM pulmonary disease while two had "indolent" NTM infection [5] that did not result in the prescription of NTM-directed antibiotic therapy. These small numbers did not allow for comparisons between those with indolent NTM infection and those with NTM pulmonary disease.

This was a single-centre study, limited by small sample size and inability to definitively determine causation despite our careful evaluation of potentially confounding clinical variables. As our study was designed to be exploratory, we chose to reduce the rate of Type II errors seen with correction for multiple comparisons in microbiome data [32], and thus cannot rule out the potential for false-positive results. While these data require validation in additional centres and cohorts, the demographics of our cohort support the generalisability of our findings. The median age of our subjects was similar to the median age of NTM infection in CF (27 years), based on the Cystic Fibrosis Foundation's (United States) patient registry data [33]. Similarly, the rate of NTM pulmonary disease diagnosis in our cohort was consistent with data from other CF centres [5]. We defined incident NTM infection using clinical AFB culture data, and acknowledge the uncertainty regarding the precise timing of the NTM infection acquisition. While AFB culture sensitivity may be limited by overgrowth of CF pathogens, and/or by sputum decontamination procedures $[34,35]$, AFB culture is more sensitive than both $16 \mathrm{~S}$ rRNA gene sequencing and quantitative PCR for NTM detection [7, 14]. Furthermore, defining incident NTM infection using sputum AFB cultures is consistent with clinical practice guidelines [3,4], and thus is a relevant approach to our ultimate goal of identifying clinically useful predictive markers of NTM pulmonary disease.

In summary, we identified features of CF airway microbiota, including relative abundances of oral-associated taxa and bacterial community interactions, preceding NTM infection and associated with clinically relevant NTM infection outcomes. As the objective of this study was to identify relationships between airway microbiota and NTM clinical course in people with CF and NTM infection, comparisons to NTM-negative people with CF was not within the scope of this study but is of interest for future studies. These data are hypothesis-generating for future studies to address knowledge gaps in our understanding of the variability in clinical outcomes of NTM infection, and to identify targets for validation as potential predictive markers of NTM pulmonary disease and novel therapeutic interventions.

Acknowledgements: This research was supported by work performed by the University of Michigan Microbial Systems Molecular Biology Laboratory. Some of the results of these studies have been reported previously at the 31st Annual North American Cystic Fibrosis Conference in 2017.

Author contributions: L.J. Caverly and J.J. LiPuma were responsible for study conception and design. L.J. Caverly, M. Zimbric, M. Azar and K. Opron contributed to acquisition, analysis and interpretation of data. L.J. Caverly drafted the manuscript, and all authors revised the manuscript for intellectual content and approved the final version.

Conflict of interest: None declared.

Support statement: This work was supported by funding from the Cystic Fibrosis Foundation to L.J. Caverly (CAVERL17A0, K-Boost Award), the National Institutes of Health (K23HL136934 to L.J. Caverly; R01HL136647-01 to J.J. LiPuma) and the University of Michigan Dept of Pediatrics Janette Ferrantino Award to L.J. Caverly. Funding information for this article has been deposited with the Crossref Funder Registry.

\section{References}

1 Bar-On O, Mussaffi H, Mei-Zahav M, et al. Increasing nontuberculous mycobacteria infection in cystic fibrosis. J Cyst Fibros 2015; 14: 53-62.

2 Adjemian J, Olivier KN, Prevots DR. Epidemiology of pulmonary nontuberculous mycobacterial sputum positivity in patients with cystic fibrosis in the United States, 2010-2014. Ann Am Thorac Soc 2018; 15: 817-826. 

consensus recommendations for the management of non-tuberculous mycobacteria in individuals with cystic fibrosis. Thorax 2016; 71: Suppl. 1, i1-i22.

4 Griffith DE, Aksamit T, Brown-Elliott BA, et al. An official ATS/IDSA statement: diagnosis, treatment, and prevention of nontuberculous mycobacterial diseases. Am J Respir Crit Care Med 2007; 175: 367-416.

5 Martiniano SL, Sontag MK, Daley CL, et al. Clinical significance of a first positive nontuberculous mycobacteria culture in cystic fibrosis. Ann Am Thorac Soc 2014; 11: 36-44.

6 Yamasaki K, Mukae H, Kawanami T, et al. Possible role of anaerobes in the pathogenesis of nontuberculous mycobacterial infection. Respirology 2015; 20: 758-765.

7 Sulaiman I, Wu BG, Li Y, et al. Evaluation of the airway microbiome in nontuberculous mycobacteria disease. Eur Respir J 2018; 52: 1800810.

8 Segal LN, Clemente JC, Li Y, et al. Anaerobic bacterial fermentation products increase tuberculosis risk in antiretroviral-drug-treated HIV patients. Cell Host Microbe 2017; 21: 530-537.

9 Mahboubi MA, Carmody LA, Foster BK, et al. Culture-based and culture-independent bacteriologic analysis of cystic fibrosis respiratory specimens. J Clin Microbiol 2016; 54: 613-619.

10 Carmody LA, Caverly LJ, Foster BK, et al. Fluctuations in airway bacterial communities associated with clinical states and disease stages in cystic fibrosis. PLoS One 2018; 13: e0194060.

11 Whiteson KL, Bailey B, Bergkessel M, et al. The upper respiratory tract as a microbial source for pulmonary infections in cystic fibrosis. Parallels from island biogeography. Am J Respir Crit Care Med 2014; 189: 1309-1315.

12 Muhlebach MS, Zorn BT, Esther CR, et al. Initial acquisition and succession of the cystic fibrosis lung microbiome is associated with disease progression in infants and preschool children. PLoS Pathog 2018; 14: e1006798.

13 Ghorbani P, Santhakumar P, Hu Q, et al. Short-chain fatty acids affect cystic fibrosis airway inflammation and bacterial growth. Eur Respir J 2015; 46: 1033-1045.

14 Caverly LJ, Carmody LA, Haig S-J, et al. Culture-independent identification of nontuberculous mycobacteria in cystic fibrosis respiratory samples. PLoS One 2016; 11: e0153876.

15 Seekatz AM, Theriot CM, Molloy CT, et al. Fecal microbiota transplantation eliminates Clostridium difficile in a murine model of relapsing disease. Infect Immun 2015; 83: 3838-3846.

16 Kozich JJ, Westcott SL, Baxter NT, et al. Development of a dual-index sequencing strategy and curation pipeline for analyzing amplicon sequence data on the MiSeq Illumina sequencing platform. Appl Environ Microbiol 2013 79: 5112-5120.

17 Westcott SL, Schloss PD. OptiClust, an improved method for assigning amplicon-based sequence data to operational taxonomic units. mSphere 2017; 2: e00073-17.

18 Nadkarni MA, Martin FE, Jacques NA, et al. Determination of bacterial load by real-time PCR using a broad-range (universal) probe and primers set. Microbiology 2002; 148: 257-266.

19 Calhoun P. 2016. Exact: Unconditional Exact Test. R Package version 17. https://cran.r-project.org/package=Exact. Date last accessed: October 2019. Date last updated: October 2019.

20 Aragon TJ. 2017. epitools: Epidemiology Tools. R Package version 05-10. https://cran.r-project.org/package= epitools. Date last accessed: October 2019. Date last updated: October 2019.

21 R: A Language and Environment for Statistical Computing. 2015. www.r-project.org. Date last accessed: October 2019. Date last updated: October 2019.

22 Ridenhour BJ, Brooker SL, Williams JE, et al. Modeling time-series data from microbial communities. ISME J 2017; 11: 2526-2537.

23 Widder S, Besemer K, Singer GA, et al. Fluvial network organization imprints on microbial co-occurrence networks. Proc Natl Acad Sci USA 2014; 111: 12799-12804.

24 Solé R, Montoya J. Complexity and fragility in ecological networks. Proc Biol Sci 2001; 268: 2039-2045.

25 Quinn RA, Whiteson K, Lim YW, et al. Ecological networking of cystic fibrosis lung infections. NPJ Biofilms Microbiomes 2016; 2: 4 .

26 Cadena AM, Ma Y, Ding T, et al. Profiling the airway in the macaque model of tuberculosis reveals variable microbial dysbiosis and alteration of community structure. Microbiome 2018; 6: 180.

27 Martiniano SL, Esther CR, Haworth CS, et al. Challenging scenarios in nontuberculous mycobacterial infection in cystic fibrosis. Pediatr Pulmonol 2020; 55: 521-525.

28 Caverly LJ, LiPuma JJ. Cystic fibrosis respiratory microbiota: unraveling complexity to inform clinical practice. Expert Rev Respir Med 2018; 12: 857-865.

29 Coburn B, Wang PW, Diaz Caballero J, et al. Lung microbiota across age and disease stage in cystic fibrosis. Sci Rep 2015; 5: 10241 .

30 Zhao J, Schloss PD, Kalikin LM, et al. Decade-long bacterial community dynamics in cystic fibrosis airways. Proc Natl Acad Sci USA 2012; 109: 5809-5814.

31 Caverly LJ, Lu J, Carmody LA, et al. Measures of cystic fibrosis airway microbiota during periods of clinical stability. Ann Am Thorac Soc 2019; 16: 1534-1542.

32 Jiang L, Amir A, Morton JT, et al. Discrete false-discovery rate improves identification of differentially abundant microbes. mSystems 2017; 2: e00092-17.

33 Adjemian J, Olivier KN, Prevots DR. Nontuberculous mycobacteria among patients with cystic fibrosis in the United States: screening practices and environmental risk. Am J Respir Crit Care Med 2014; 190: 581-586.

34 Bange FC, Böttger E. Improved decontamination method for recovering mycobacteria from patients with cystic fibrosis. Eur J Clin Microbiol Infect Dis 2002; 21: 546-548.

35 Buijtels PCAM, Petit PLC. Comparison of NaOH-N-acetyl cysteine and sulfuric acid decontamination methods for recovery of mycobacteria from clinical specimens. J Microbiol Methods 2005; 62: 83-88. 